\title{
Liposuction: the way I've lived it
}

\section{Introduction}

I heard about this "new" procedure in the late 70's, when the opinion was divided between safety or dangerousness, as well as its efficacy or lack of it. Either way, I continue learning about it, looking on the form to make it much more effective and certainly safer. But probably the way to learn more on a procedure is performing many cases, taking out the most important aspects of it and talks about it in meetings with surgeons working in the same direction. By that time, I was a speaker two or three times a year into the nicest meetings organized in the Graduate Hospital of Philadelphia with Julius Newman, and with Garry Fenno in Houston. On those days the discussion were mainly oriented to the volumes of aspiration, blood losses and the type of anesthesia used, although a majority of colleagues were decanted toward the general one. ${ }^{1-3}$ Certainly Liposuction was the main vehicle what made flourish the Cosmetic Surgery and some related procedures like Fat transfer, this happened to be the motor who promoted the birth of the American Academy of Cosmetic Surgery, the Meso American Academy of Cosmetic Surgery, and the American Board of Cosmetic Surgery, which I approved on 1990. At that time also I could be witness of the first lecture on tumescent anesthesia. On 1986 I was ready to present a talk on Lipo at the Graduate Hospital in Philadelphia sitting between Pierre Fournier and Richard Webster, when was the turn of a young dermatologist who presented his new concept of tumescent anesthesia; with this 15 minutes talk, Jeffrey Klein entered into the history and changed the panorama not only of

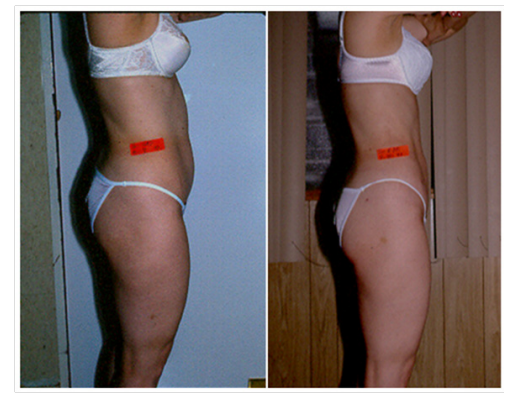

Figure I A middle age lady with only minor fat deposits in the abdomen. A nice case for liposuction before and after.

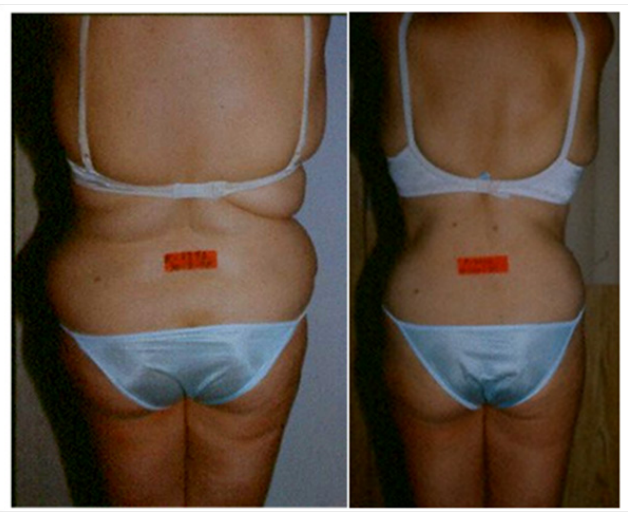

Figure 3 A middle age Lady with large fat deposits in waist, hips and lower back, before and after.
Volume 2 Issue 2 - 2018

\author{
Enrique Hernández Pérez,' José Enrique \\ Hernández Pérez, ${ }^{2}$ Mauricio Hernández \\ Pérez ${ }^{2}$ \\ 'Director, Center for Dermatology and Cosmetic Surgery, El \\ Salvador \\ ${ }^{2}$ Associated Professor, Center for Dermatology and Cosmetic \\ Surgery, El Salvador
}

Correspondence: Enrique Hernández Pérez MD, Director Center for Dermatology and Cosmetic Surgery, El Salvador, Email drenrique@hernandezperez.com

Received: January 14, 2018 | Published: Aprial 02, 2018

Lipo but also Cosmetic Surgery. ${ }^{1-3}$

\section{Lipo: How we perform it}

Based upon more than 35years' experience and many cases operated getting excellent results (up to 6cases in a single day), these are the protocol we follow in our cases (Figures 1-3).

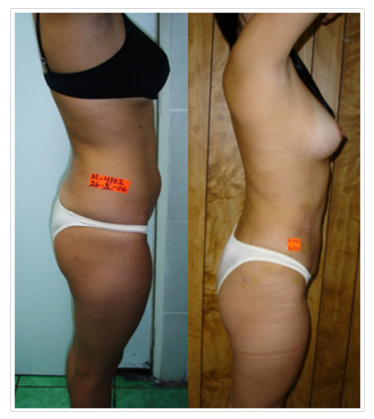

Figure 2 A young girl with small fat deposits in the abdomen and waist. Good case for Liposuction. Before and after.

\section{Preparation of the case}

Patients must be clearly explained that end results will depend of an exact attachment to a careful diet and physical exercise starting soon after surgery and keeping so from here on.

All the patients are submitted to a careful physical examination, as well as cardiovascular exam, demanding a written statement by the cardiologist allowing us the use of epinephrine in the local anesthesia. ${ }^{2-4}$ An oral antibiotic is started 3 days before and kept along a total of 5 days. The day before patient is instructed to use a local antiseptic in the shower; our favorites: povidone iodine or clorhexidine nitrate. ${ }^{2,3}$

\section{Anesthesia}

Although we have more than 15years of not using general anesthesia, in order to avoid unpleasant memories regarding surgery, 
a certified anesthesiologist provides IV sedation: the most

common drugs are midazolam, fentanyl and Propofol. ${ }^{4}$ Regarding local anesthesia, we continue being faithful to the Klein tumescent anesthesia. Our preparation, made at the moment, is very simple: At 1 liter of saline solution we add $25 \mathrm{cc}$ of Lidocaine plus $1 \mathrm{cc}$ of $1 \%$ epinephrine. We avoid the use of corticoids or sodium bicarbonate in the preparation because they provoke shorten in the analgesia period and increase the inflammation. ${ }^{4}$

\section{Estimating the amount of anesthesia}

This keeps close relationship with the volume to be aspirated. In the present time we are not performing large volumes lipo, as we did in the past. ${ }^{2,4}$ In general; we prefer not to exceed 6 liters aspiration in a single session. If the case requires much more, we prefer to program 2 or 3 sessions, one week apart to each other. This way the recovery is easier. To estimate the amount of safe anesthesia, we take the weight in $\mathrm{kg}$ of the patient and multiply by 7; then approximate this figure to the nearest upper figure, and multiply by 1.5 that is a constant. Table 1 shows some common cases and our approach for that. ${ }^{2,4}$ This amount in liters is only a very far limit or roof; the precaution tell us never to reach that figure, due to safety reasons.

Table I For getting the figures at right, approximate to the upper closest one, for instance: $490=5$, then multiply by the constant I,5. This way, we can use up to 7.5Liters of Klein solution. Modified From Hernández-Pérez E: Temas Selectos en Cirugía Cosmética²

\begin{tabular}{lll}
\hline Correlating weight $\mathbf{( K g )}$ & Units & Litres to infiltrate \\
\hline 70 & 490 per I,5 & 7.5 \\
80 & 560 & 9 \\
90 & 630 & 9
\end{tabular}

At the end the aspiration is very easy, handling cannulas no greater than $3 \mathrm{~mm}$. But remember, the thinner, the better. If we want the fat for posterior grafting, we use syringes instead of cannulas. They preserve better the delicate adipocytes. And be careful: Surgery must be learned next to experienced surgeons. ${ }^{4}$ At the end, starting one week thereafter, we perform 5 sessions of ultrasound or radiofrequency, weekly, in order to stretch the skin, avoiding irregularities and becoming smoother. We prefer this simple way instead of laser lipo as used by other much respected colleagues. ${ }^{5-7}$

But take care and proceed very cautiously. When well performed, Liposuction surgery ought to be a surgery lacking complications. ${ }^{8,9}$

\section{Conclusion}

In summary, we finish giving all sorts of advices as an outcome from many years of experience (Table 2).
Table 2 Temas Selectos en Cirugía Cosmética ${ }^{2}$

\section{Rules that invites to a disaster (must avoid)}

Try to walk by roads you don't fully know

Condemn any procedure, no matter how good might it be, if have been originated in another specialty

To save money for a patient, try to combine several procedures at the same time

Try to work in Operating Rooms that are not fully equipped

Take risks out of reasonable limits

\section{Acknowledgements}

None.

\section{Conflicts of interest}

The authors declared that there are no conflicts of interest

\section{References}

1. Klein JA. Tumescent technique. Tumescent Anesthesia \& Microcannular Liposuction. $1^{\text {st }}$ ed. St Louis: Mosby; 2000.

2. Hernández Pérez E. Temas Selectos en Cirugía Cosmética. $1^{\text {st }}$ ed. Odontomedi México: Mexico; 2012. 365 p.

3. Shiffman MA, Di Giusepe A. Liposuction, principles and practice. New York: Springer; 2006. 568 p.

4. Hernández-Pérez E, Lozano E. Volume Liposculture. Variations on technique. Cosmet Dermatol. 1999;12:35-39.

5. Matarasso A, Levine SM. Evidence-based medicine: liposuction. Plast Reconstr Surg. 2014;132(6):1697-1705.

6. Collins PS, Moyer KE. Evidence-Based Practice in Liposuction. Ann Plast Surg. 2018.

7. Pereira-Netto D, Montano-Pedroso JC, Aidar ALES, et al. LaserAssisted Liposuction (LAL) Versus Traditional Liposuction: Aesthetic Plast Surg. 2018;42(2):376-383.

8. Hanke CW, Bernstein G, Bullock S. Safety of tumescent liposuction in 15,336 patients. National Survey Results. Dermatol Surg. $1995 ; 21(5): 459-462$.

9. Ezzeddine H, Husari A, Nassar H, et al. Life Threatening Complications Post-Liposuction. Aesthethic Plast Surg. 2018;42(2):384-387. 